\title{
Radiotherapy plus concurrent and adjuvant procarbazine, lomustine, and vincristine chemotherapy for patients with malignant glioma
}

\author{
SEUNG-HO YANG ${ }^{1}$, YONG-KIL HONG ${ }^{1}$, SEI-CHUL YOON ${ }^{2}$, BUM-SOO KIM ${ }^{3}$, YOUN-SOO LEE ${ }^{4}$, \\ TAE-KYU LEE ${ }^{1}$, KWAN-SUNG LEE ${ }^{1}$, SIN-SOO JEUN ${ }^{1}$, MOON-CHAN KIM ${ }^{1}$ and CHUN-KUN PARK ${ }^{1}$ \\ Departments of ${ }^{1}$ Neurosurgery, ${ }^{2}$ Radiation Oncology, ${ }^{3}$ Radiology, and ${ }^{4}$ Hospital Pathology, \\ Kangnam St. Mary's Hospital, The Catholic University of Korea, Seoul, Korea
}

Received January 9, 2007; Accepted March 8, 2007

\begin{abstract}
We analyzed the clinical efficacy and toxicity of concurrent therapy as a first line modality for malignant glioma patients. From 1998 to 2004, 39 patients, 22 with glioblastoma (GM), nine with anaplastic astrocytoma (AA), 7 with anaplastic oligodendroglioma (AO) and 1 with anaplastic oligodendro-astrocytoma (AOA) were enrolled in this study. The median age was 46.2 years (range 8-67). Both external involved field radiotherapy and chemotherapy, composed of CCNU $\left(75-110 \mathrm{mg} / \mathrm{m}^{2}\right)$, procarbazine $\left(60 \mathrm{mg} / \mathrm{m}^{2}\right)$ and vincristine $\left(1.4 \mathrm{mg} / \mathrm{m}^{2}\right)$, were started simultaneously two weeks after surgery. The median progression-free survival time for the GM, AA, and AO patients was 6, 26, and 31 months, respectively. The median survival of the patients with GM and AA was 27 and 41 months. The two-year survival rate of the GM and AA patients was 50.4 and $66.7 \%$, respectively. Grade III/IV hematological toxicity was reduced from 25.6 to $13 \%$ after reduction of the dose of CCNU $\left(75 \mathrm{mg} / \mathrm{m}^{2}\right)$. Radiation necrosis was confirmed by pathologic examination in four patients $(10.3 \%)$. The median interval from the completion of radiotherapy to the diagnosis of necrosis was 19 weeks. Modified concurrent chemoradiotherapy may be a feasible option for treating malignant glioma with acceptable toxicity.
\end{abstract}

\section{Introduction}

The prognosis of patients with malignant glioma remains poor despite advances in neurosurgery, radiotherapy and chemotherapy. Studies have confirmed the beneficial effect of postoperative cranial irradiation, but with a prolongation

Correspondence to: Dr Yong-Kil Hong, Department of Neurosurgery, Kangnam St. Mary's Hospital, The Catholic University of Korea, 505 Banpo-dong, Seocho-gu, Seoul 137-701, Korea

E-mail: hongyk@catholic.ac.kr

Key words: chemotherapy, combined modality therapy, glioma, radiation necrosis, toxicity of median survival of nine to 12 months $(1,2)$. The role of adjuvant chemotherapy for these patients has been less apparent. Two meta-analyses $(3,4)$ have evaluated the use of adjuvant chemotherapy in patients with malignant glioma. They suggested that patients treated with radiotherapy plus adjuvant chemotherapy had a survival advantage, regardless of several prognostic factors such as histology, age, performance status and extent of disease. In these reports, the survival benefit associated with nitrosourea-based adjuvant chemotherapy regimens was modest, but highly significant.

There are several reports on concurrent chemo-radiotherapy in patients with malignant glioma (5-8). It has been postulated that concurrent chemo-radiotherapy takes advantage of the complementary effects of both therapies. Radiation recruits $G_{0}$ cells into the $G_{1}$ phase of the cell cycle, the phase most susceptible to many chemotherapeutic agents. The use of two different therapies with a different spectrum of toxicity may enable the eradication of cell lines resistant to a single therapy (9). Chemotherapy may block cellular repair mechanisms preventing recovery from sublethal radiation damage (10), while radiation may facilitate entry into the central nervous system impermeable to cytotoxic drugs by disrupting the blood-brain barrier.

Although recent studies have focused on the new alkylating and methylating agent, temozolomide, for treatment of malignant glioma, the role of procarbazine, lomustine, and vincristine (PCV) chemotherapy has been shown to be a matter of debate in adjuvant settings (11-14). In this study, we report the efficacy and toxicity of concurrent modified PCV chemo-radiotherapy. Major complications of the concurrent therapy, hematotoxicity and radiation necrosis, were analyzed.

\section{Patients and methods}

Eligibility criteria. Patients treated at a single institution in Korea between 1998 and 2004 with newly diagnosed malignant glioma were identified. The histological diagnosis of malignant glioma was based on the World Health Organization (WHO) classification. They were offered the concurrent PCV chemo-radiotherapy if the following criteria were met: Karnofsky performance status (KPS) scores of $\geq 70$ 
Table I. Baseline characteristics of patients.

\begin{tabular}{lc}
\hline Sex & \\
Male & 21 \\
Female & 18 \\
Age, median (range) & 46.2 years (8-67) \\
Pathology & \\
Glioblastoma multiforme & $22(56.4 \%)$ \\
Anaplastic astrocytoma & $9(23.1 \%)$ \\
Anaplastic oligodendroglioma & $7(17.9 \%)$ \\
Anaplastic oligoastrocytoma & $1(2.6 \%)$ \\
Extent of surgery & $13(33.3 \%)$ \\
Gross total resection & $16(41 \%)$ \\
Partial resection & $10(25.7 \%)$ \\
Biopsy & $1.7(1-4)$ \\
Cycles of chemotherapy, mean (range) & $5400-5940 \mathrm{cGy}$ \\
Dose of radiotherapy
\end{tabular}

on postoperative day 14 , adequate bone marrow reserve (leukocyte count $>4000 \mathrm{~mm}^{3}$ and platelet $>150,000 \mathrm{~mm}^{3}$ ), normal baseline liver (serum bilirubin level $<20 \mathrm{mmol} / \mathrm{l}$ ), renal (serum creatinine level $<150 \mathrm{mmol} / \mathrm{l}$ ) and cardiac function, no previous chemotherapy or radiotherapy, and no known medical or psychiatric history. Voluntary, informed, written consent was obtained from all patients.

Therapeutic protocol. Chemotherapy and radiotherapy were started on day 14 postoperatively. All patients underwent involved field partial brain irradiation, and the tumor volume for irradiation was determined by defining the volume of the contrast-enhancing tumor itself and $\sim 2-\mathrm{cm}$ margins beyond the edema surrounding the tumor by postoperative magnetic resonance images (MRI). The radiation therapy was carried out by a 6-MV linear acceleration (180 cGy/day for 5 days each week), totaling 5400-5940 cGy. Concurrent chemotherapy included $110 \mathrm{mg} / \mathrm{m}^{2}$ of CCNU (or $120 \mathrm{mg} / \mathrm{m}^{2}$ of BCNU), $60 \mathrm{mg} / \mathrm{m}^{2}$ of procarbazine and $1.4 \mathrm{mg} / \mathrm{m}^{2}$ of vincristine simultaneously started with the radiotherapy. Briefly, CCNU (or BCNU) was administered $1 \mathrm{~h}$ before radiotherapy on day 1 . Vincristine was infused $30 \mathrm{~min}$ before radiotherapy on day 8 and 28. Procarbazine was administered from day 8-21. The subsequent cycle of adjuvant chemotherapy was followed four weeks after the last radiotherapy treatment. From 2002, enrolled patients received a reduced dose of CCNU $\left(75 \mathrm{mg} / \mathrm{m}^{2}\right)$ in the first cycle and the usual dose in subsequent cycles. This change was made due to the moderate to severe hematological toxicity experienced by the patients during the early period of the study. The dose of CCNU was also modified during the subsequent cycles when grade III/IV leukocytopenia or thrombocytopenia occurred. Hematotoxicity was classified according to the National Cancer Institute Toxicity Criteria (15).

Statistical analysis. The study end points of this trial were overall survival (OS) and progression-free survival (PFS). PFS was measured from the start of the concurrent therapy to

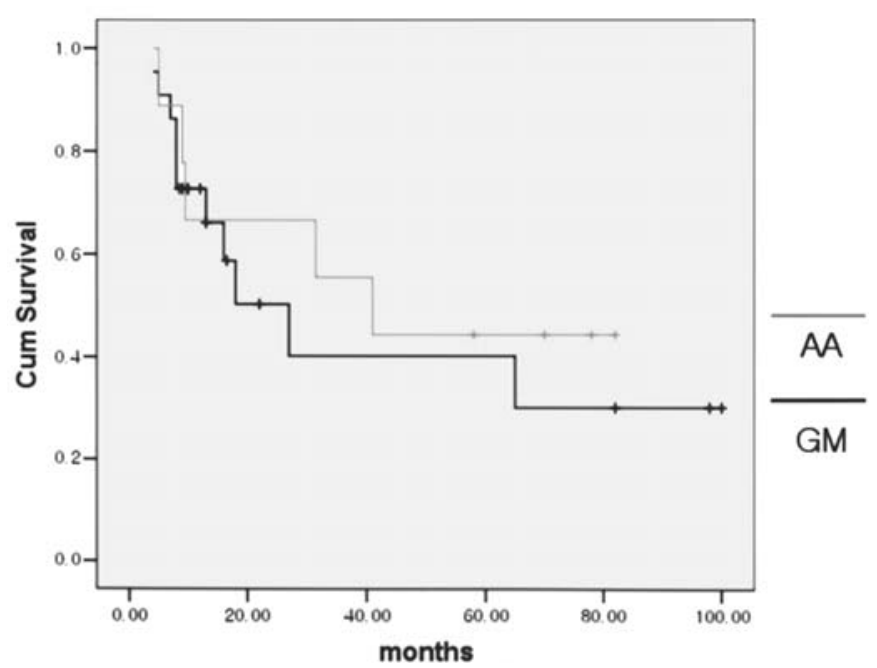

Figure 1. Kaplan-Meier overall survival in glioblastoma (GM) and anaplastic astrocytoma (AA) patients.

tumor progression or to withdrawal from the study. OS was calculated from the date of histological diagnosis to death or to the last date of follow-up. Progression or relapse was defined as growth or regrowth of tumor in the central nervous system. The Kaplan-Meier method was used to estimate OS and PFS using SPSS software (version 11.0, Chicago, IL).

\section{Results}

Characteristics of patients. From 1998 to 2004, 39 patients were treated with concurrent chemotherapy and radiotherapy according to the protocol guidelines. They included 22 glioblastoma (GM), 9 anaplastic astrocytoma (AA), 7 anaplastic oligodendroglioma $(\mathrm{AO})$ and 1 anaplastic oligodendro-astrocytoma (AOA) patients. The median patient age was 46.2 years (range 8-67). The total number of cycles of chemotherapy was 67 with a mean of 1.7 cycles administered (range, 1-4 cycles). The median follow-up period was 18 months (range, 5-100 months). The main clinical features are summarized in Table I.

Progression-free survival and overall survival. All patients completed the concurrent chemo-radiotherapy. If tolerated without clinical deterioration, subsequent adjuvant PCV chemotherapy was administered. Fifteen patients $(38.5 \%)$ received the subsequent $\mathrm{PCV}$ chemotherapy after the concurrent chemo-radiotherapy.

The median PFS of GM, AA, and AO patients was 6, 26, and 31 months, respectively. When progression or relapse of disease was noted, salvage chemotherapy was administered. Eighteen patients (11 GM, 5 AA, and 2 AO) with tumor progression were treated with temozolomide chemotherapy.

The median survival of GM and AA patients was 27 months (95\% CI 11.1-42.9) and 41 months (95\% CI 13.2$68.8)$, respectively. The one-year and two-year survival rates of GM patients were 72.7 and $50.4 \%$. The two-year survival rate of AA was $66.7 \%$. The median survival of AO patients has not yet been obtained. The Kaplan-Meier survival curve is presented in Fig. 1. 
Table II. Toxicity according to the National Cancer Institute Toxicity Criteria.

\begin{tabular}{lcccc}
\hline & \multicolumn{3}{c}{ Grade } & IV $(\%)$ \\
\cline { 2 - 5 } & I (\%) & II $(\%)$ & III $(\%)$ & $2(5.1)$ \\
\hline Leukopenia & $4(10.3)$ & $3(7.7)$ & $6(15.4)$ & - \\
Thrombocytopenia & $3(7.7)$ & $3(7.7)$ & $2(5.1)$ & $1(2.6)$ \\
Nausea/vomiting & $5(12.8)$ & $3(7.7)$ & $2(5.1)$ & $1(2.6)$ \\
Hepatic & - & $1(2.6)$ & &
\end{tabular}

A

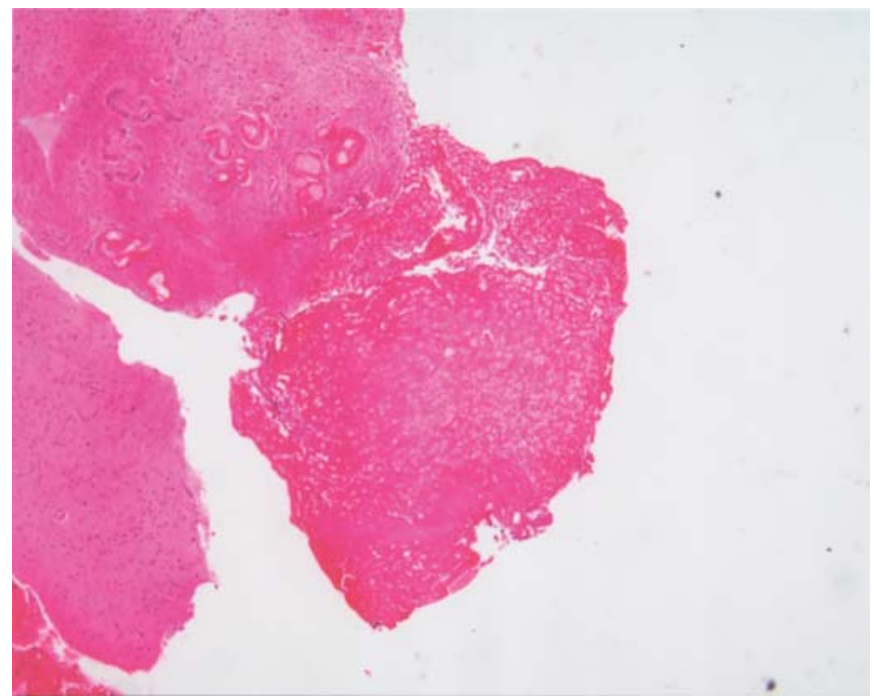

B

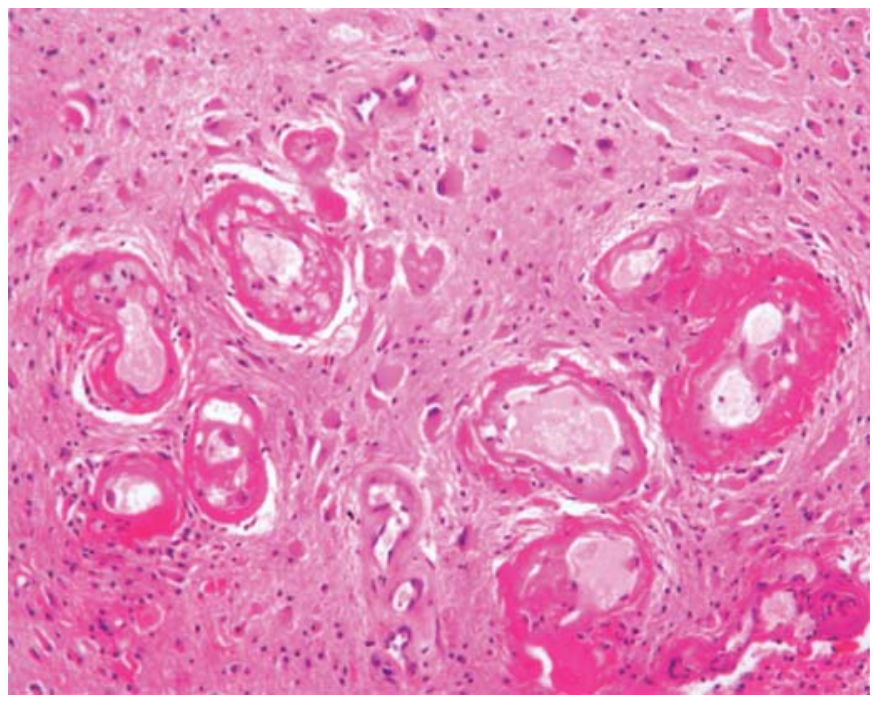

Figure 2. Histological features of radiation necrosis. (A) Area of extensive necrosis in the brain parenchyma (H\&E, $\mathrm{x} 40$ ). (B) Hyalinizing thickening of vascular wall and surrounding reactive astrocytes having reactive radiation atypia (H\&E, x200).

Table III. Characteristics of patients diagnosed with radiation necrosis after concurrent radio-chemotherapy.

\begin{tabular}{|c|c|c|c|c|c|c|c|c|}
\hline Sex & Age & Pathology & $\begin{array}{c}\text { Clinical } \\
\text { manifestation }\end{array}$ & $\begin{array}{c}\text { Chemotherapy } \\
\text { (cycle) }\end{array}$ & $\begin{array}{c}\text { Radiotherapy } \\
\text { (cGy) }\end{array}$ & Diagnosis & $\begin{array}{l}\text { Interval }^{\mathrm{a}} \\
\text { (week) }\end{array}$ & $\begin{array}{c}\text { OS } \\
\text { (month) }\end{array}$ \\
\hline Female & 53 & GM & Headache & PBV (x2) & 5940 & Resection & 46 & $82.0^{+}$ \\
\hline Male & 8 & $\mathrm{AA}$ & Headache & PCV (x3) & 5500 & Biopsy & 16 & $78.0^{+}$ \\
\hline Female & 39 & $\mathrm{AO}$ & Headache & $\operatorname{PCV}(x 2)$ & 5580 & Resection & 10 & 49.5 \\
\hline Female & 28 & $\mathrm{AO}$ & Hemiparesis & PCV (x1) & 5940 & Resection & 8 & 23.0 \\
\hline
\end{tabular}

GM, glioblastoma multiforme; AA, anaplastic astrocytoma; AO, anaplastic oligodendroglioma. PCV, (procarbazine + CCNU + vincristine);

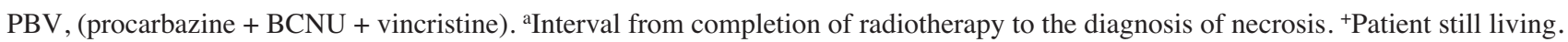

Toxicity. Several toxicities were noted including gastrointestinal problems, hematological toxicities, radiation-induced necrosis, and pneumonia. One patient died from leukopenia followed by severe pneumonia.

Blood counts were evaluated at least once a week during concurrent therapy. The most frequent toxicity was leukopenia which developed in 15 patients $(38.5 \%)$. Grade III/IV hematological toxicity was present in $25.6 \%$ (10 patients). After the administration of reduced CCNU $\left(75 \mathrm{mg} / \mathrm{m}^{2}\right)$, grade
III/IV hematological toxicity was decreased to $13 \%$ (3 out of 22 patients). Leukocytopenia and thrombocytopenia developed simultaneously in five patients. Nonhematological toxicity included mainly hepatic or gastrointestinal problems; these patients were treated with conservative care and did not drop out of the trial. Treatment-related toxicities are outlined in Table II.

In four patients $(10.3 \%)$, radiation necrosis was confirmed by pathologic examination (Fig. 2). Their characteristics are 
A

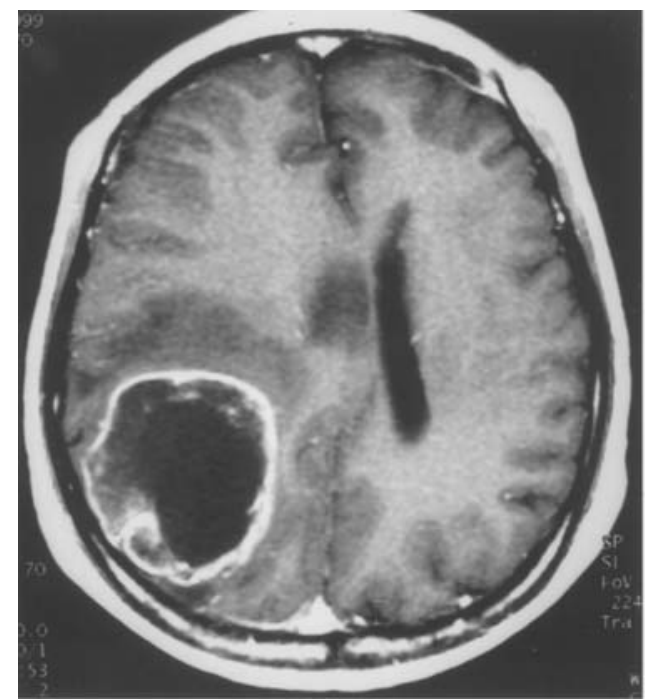

$\mathrm{C}$

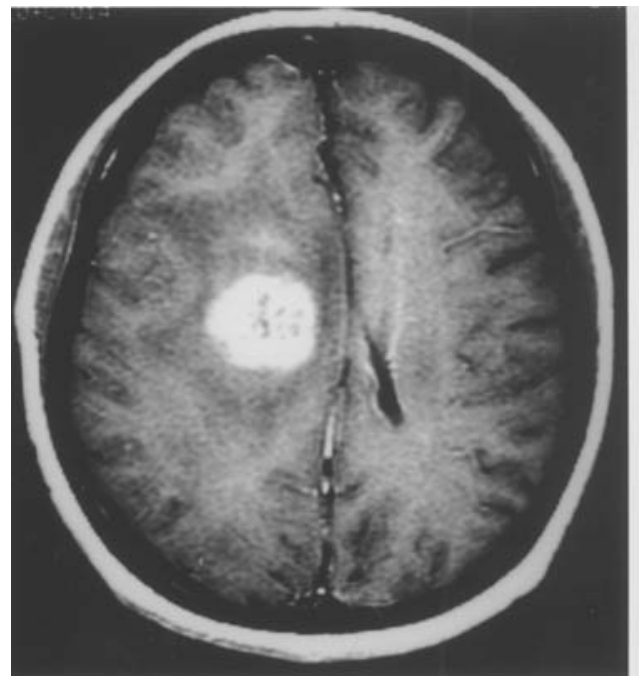

B



$\mathrm{D}$



Figure 3. A case showing a cystic mass in preoperative T1-weighted gadolinium-enhanced axial MRI (A). The homogenous enhancing lesion (B) developed after concurrent chemo-radiotherapy was diagnosed with radiation necrosis. Another case showing the enhancing lesion (C), preoperatively. After concurrent therapy, cystic change (D) developed, and radiation necrosis was confirmed by the resection.

summarized in Table III. Clinical deterioration with headache and hemiparesis led us to perform a radiological examination. The interval from the initial date of irradiation to the detection of radiation necrosis was $6,8,16$, and 46 weeks, respectively. After resection, the symptoms were relieved and then the patients were observed without adjuvant therapy. Fig. 3 shows the magnetic resonance images before and after concurrent therapy. The enhancing lesions mimic tumor progression. Two patients with glioblastoma and anaplastic astrocytoma showed a longer survival; 82 and 78 months, respectively and are still alive.

\section{Discussion}

The PCV combination regimen has been extensively used for the treatment of malignant glioma. The results of previous trials suggest that PCV chemotherapy is still inadequate $(16,17)$. Murphy et al reported concurrent chemo-radiotherapy using PCV regimen in 27 patients with GM and a median OS of 20.5 months (18). Recently, Brandes et al reported no significant difference in terms of progression and survival between PCV and temozolomide treatment after surgery and radiation in patients with AA (19). In the PCV group, 83 and $74 \%$ of patients were alive at two and three years, respectively. In our study, the median survival of GM and AA patients was 27 and 41 months, respectively. The two-year survival rate of AA patients was $66.7 \%$. The relatively longer survival of our patients appears to be influenced by the good performance status before surgery and the young age of participants (median 46.2 years); the $\mathrm{PCV}$ regimen is not easy to tolerate, especially in elderly patients. In addition, salvage temozolomide chemotherapy for recurrence and progression may have partially contributed to the longer observed survival.

Because of a high incidence of hematological toxicity with the radiotherapy and concurrent PCV chemotherapy, the regimen was modified. In the early period of our trial, grade III/IV hematological toxicities occurred in $25.6 \%$ of the patients and delayed the treatment schedules. For this reason, the dose of CCNU was reduced by $30 \%$. Afterwards, grade 
III/IV toxicities were significantly decreased to $13 \%$, and subsequent hospital admission was not necessary. The most common adverse event, hematological toxicity, usually occurred between the fourth and eighth week after the administration of CCNU. Therefore, hematological surveillance must be continued after the completion of chemoradiotherapy.

The distinction between radiation necrosis and recurrent malignant glioma remains a challenge despite the use of advanced imaging techniques such as perfusion- and diffusionweighted MR imaging $(20,21)$, MR spectroscopy (22), and positron emission tomography (23). In our study, the pathological diagnosis of radiation necrosis was determined in four patients $(10.3 \%)$ by the removal of symptom-related lesions. The mean interval from the completion of radiotherapy to the diagnosis of necrosis was 19 weeks. In one study (24), cerebral radionecrosis was diagnosed with an incidence of $4.8 \%$; the mean latent interval from completion of radiotherapy with or without adjuvant chemotherapy to the diagnosis of necrosis was 11.6 months (range, 2-32 months). Through this study the authors demonstrated that the use of adjuvant chemotherapy was strongly related to subsequent development of necrosis. Peterson et al reported an incidence of radionecrosis of $2.5 \%$ ranging from $8-31$ months after radiotherapy in 200 patients treated with sequential radiotherapy and chemotherapy (25). Our results showed that the concurrent chemo-radiotherapy increased the risk of subsequent necrosis early after the completion of radiotherapy. Concomitant temozolomide and radiotherapy are now considered standard care for the treatment of GM (26). Therefore, an increase in the incidence of radiation necrosis may be expected in the future. The transient increase in apparent tumor volume shortly after the end of radiotherapy is referred to as pseudoprogression (27). However, the difference between radiation necrosis and pseudoprogression has not yet been defined.

Radiation necrosis is known to be associated with considerable morbidity and mortality (28). However, the findings of some studies suggest that the outcomes in patients with malignant glioma who survive after the development of radiation necrosis may actually be more favorable than in those who do not develop this complication $(29,30)$. In our study, 3 patients with radiation necrosis survived longer compared to patients with the same pathology. Even though the number of enrolled patients was small and statistical analysis was not available, 2 patients with GM and AA, diagnosed with radiation necrosis after concurrent therapy, are alive 82 and 78 months after the first surgery. This finding suggests that much of the necrosis identified in this setting was not necrosis of normal tissue, but rather the tumor itself.

We addressed the question of whether radiation necrosis is associated with the clinical prognosis and how patients cope with it. Although spontaneous resolution may be obtained after corticosteroid therapy alone in some cases, we suggest that the resection of symptom-related lesions should be considered, not only for differentiating tumor progression, but also for the relief of symptoms such as headache, hemiparesis, or change of mental status.

In conclusion, modified PCV chemo-radiotherapy is a feasible option for patients with malignant glioma. It is effective with acceptable toxicity. More frequent and earlier detection of radiation necrosis, during the concurrent chemoradiotherapy can be expected in the future. If the suspicious lesions are symptomatic, surgical resection is recommended for precise determination and patient management.

\section{References}

1. Walker MD, Alexander E, Hunt WE, et al: Evaluation of BCNU and/or radiotherapy in the treatment of anaplastic gliomas. J Neurosurg 49: 333-343, 1978.

2. Kristiansen K, Hagen S, Kollevold T, et al: Combined modality therapy of operated astrocytomas grade III and IV: confirmation of the value of postoperative irradiation and lack of potentiation of bleomycin on survival time. Cancer 52: 997-1007, 1983.

3. Fine HA, Dear KB, Loeffler JS, Black PM and Canellos GP: Meta-analysis of radiation therapy with and without adjuvant chemotherapy for malignant gliomas in adults. Cancer 71: 2585-2597, 1993.

4. Stewart LA: Chemotherapy in adult high-grade glioma: a systemic review and meta-analysis of individual patient data from 12 randomized trials. Lancet 359: 1011-1018, 2002.

5. Yung WKA, Janus TJ, Maor M and Feun LG: Adjuvant chemotherapy with carmustine and cisplatin for patients with malignant gliomas. J Neurooncol 12: 131-135, 1992.

6. Chamberlain MC, Barba D, Lormanik P, Berson AM, Saunders WM and Shea MC: Concurrent cisplatin therapy and iodine 125 brachy-therapy for recurrent malignant brain tumors. Arch Neurol 52: 162-167, 1995.

7. Brandes AA, Rigon A, Zampieri P, Ermani M, Carollo C and Altavilla G: Carboplatin and teniposide concurrent with radiotherapy in patients with glioblastoma multiforme: a phase II study. Cancer 82: 355-361, 1998.

8. Kleinberg L, Grossman SA, Piantadosi S, Zeltzman M and Wharam M: The effects of sequential versus concurrent chemotherapy and radiotherapy on survival and toxicity in patients with newly diagnosed high-grade astrocytoma. Int J Radiat Oncol Biol Phys 44: 535-543, 1999.

9. Vokes EE and Wechselbaum R: Concomitant chemoradiotherapy: rationale and clinical experience in patients with solid tumors. J Clin Oncol 8: 911-934, 1990.

10. Brandes AA, Rigon A, Zampieri P, et al: Early chemotherapy and concurrent radio-chemotherapy in high grade glioma. J Neurooncol 30: 247-255, 1996.

11. Kappelle AC, Postma TJ, Taphoorn MJ, et al: PCV chemotherapy for recurrent glioblastoma multiforme. Neurology 56: 118-120, 2001.

12. Levin VA, Hess KR, Choucair A, et al: Phase III randomized study of postradiotherapy chemotherapy with combination alpha-difluoromethylornithine-PCV versus PCV for anaplastic gliomas. Clin Cancer Res 9: 981-990, 2003.

13. Triebels VH, Taphoorn MJ, Brandes AA, et al: Salvage PCV chemotherapy for temozolomide-resistant oligodendrogliomas. Neurology 63: 904-906, 2004.

14. Schmidt F, Fischer J, Herrlinger U, Dietz K, Dichgans J and Weller M: PCV chemotherapy for recurrent glioblastoma. Neurology 66: 587-589, 2006.

15. Miller AB, Hoogstraten B, Staquet M and Winkler A: Reporting results of cancer treatment. Cancer 47: 207-214, 1981.

16. Prados M, Scott C, Curran W, Nelson D, Leibel S and Kramer S: Procarbazine, lomustine, and vincristine (PCV) chemotherapy for anaplastic astrocytoma: a retrospective review of radiation therapy oncology group protocols comparing survival with carmustine or PCV adjuvant chemotherapy. J Clin Oncol 17: 3389-3395, 1999.

17. The Medical Research Council Brain Tumour Working Party: Randomized trial of procarbazine, lomustine, and vincristine in the adjuvant treatment of high-grade astrocytoma: a medical research council trial. J Clin Oncol 19: 509-518, 2001.

18. Murphy C, Pickles C, Knowling M and Thiesse B: Concurrent modified PCV chemotherapy and radiotherapy in newly diagnosed grade IV astrocytoma. J Neurooncol 57: 215-220, 2002.

19. Brandes AA, Nicolardi L, Tosoni A, et al: Survival following adjuvant PCV or temozolomide for anaplastic astrocytoma. Neurooncol 8: 253-260, 2006.

20. Biousse V, Newman NJ, Hunter SB and Hudgins PA: Diffusionweighted imaging in radiation necrosis. J Neurol Neurosurg Psychiatry 74: 382-384, 2003. 
21. Hein PA, Eskey CJ, Dunn JF and Hug EB: Diffusion-weighted imaging in the follow-up of treated high-grade gliomas: tumor recurrence versus radiation injury. Am J Neuroradiol 25: 201-209, 2004.

22. Schlemmer HP, Bachert P, Henzae M, et al: Differentiation of radiation necrosis from tumor progression using proton magnetic resonance spectroscopy. Neuroradiology 44: 216-222, 2002.

23. Chao ST, Suh JH, Raja S, Lee SY and Barnett G: The sensitivity and specificity of FDG PET in distinguishing recurrent brain tumor from radionecrosis in patients treated with stereotactic radiosurgery. Int J Cancer 96: 191-197, 2001.

24. Ruben JD, Dally M, Bailey M, Smith R, McLean CA and Fedele P: Cerebral radiation necrosis: incidence, outcome, and risk factors with emphasis on radiation parameters and chemotherapy. Int J Radiat Oncol Biol Phys 65: 499-508, 2006.

25. Peterson K, Clark B, Hall W and Truwit CL: Multifocal enhancing magnetic resonance imaging lesions following cranial irradiation. Ann Neurol 38: 237-244, 1995.
26. Stupp R, Mason WP, van den Bent MJ, et al: Radiotherapy plus concomitant and adjuvant temozolomide for glioblastoma. $\mathrm{N}$ Engl J Med 352: 987-996, 2005.

27. de Wit MC, de Bruin HG, Eijkenboom W, Sillevis Smitt PA and van den Bent MJ: Immediate postradiotherapy changes in malignant glioma can mimic tumor progression. Neurology 63: 535-537, 2004.

28. Sheline GE, Wara WM and Smith V: Therapeutic irradiation and brain injury. Int J Radiat Oncol Biol Phys 6: 1215-1228, 1980.

29. Forsyth PA, Kelly PJ, Cascino TL, et al: Radiation necrosis or glioma recurrence: is computer-assisted stereotactic biopsy useful? J Neurosurg 82: 436-444, 1995.

30. Floyd NS, Woo SY, Teh BS, et al: Hypofractionated intensitymodulated radiotherapy for primary glioblastoma multiforme. Int J Radiat Oncol Biol Phys 58: 721-726, 2004. 